\title{
On the Critical Temperature of Non-Periodic Ising Models on Hexagonal Lattices
}

\author{
Ferenc Iglói and Péter Lajkó \\ Research Institute for Solid State Physics \\ H-1525 Budapest, P.O.Box 49, Hungary \\ and \\ Institute for Theoretical Physics, Szeged University \\ H-6720 Szeged, Aradi V. tere 1, Hungary
}

\begin{abstract}
The critical temperature of layered Ising models on triangular and honeycomb lattices are calculated in simple, explicit form for arbitrary distribution of the couplings.
\end{abstract}

PACS-numbers: 05.50.+q, 64.60.Cn, 64.60.Fr 
In recent years we have witnessed a growing theoretical interest to understand the critical properties of non-periodic (quasi-periodic, aperiodic or random) systems[1-4]. Periodic systems, which are built of regularly spaced blocks or unit cells, are assumed to share the critical properties of the homogeneous model. Indeed close to the critical point the correlation length is much larger than the linear size of the blocks, thus averaging over correlated domains the periodic system becomes homogeneous. This argument naturally fails, if the size of the unit cell is diverging, i.e. for non-periodic models. In these systems - in the spirit of the Harris criterion[5] - one should investigate the fluctuations of the couplings in a domain of correlated spins. The perturbation, caused by the non-periodicity is then irrelevant (relevant), if the local energy fluctuations are smaller (greater) than the corresponding thermal energy.

In study the critical behaviour of specific systems it is very important to know the position of the critical point exactly, since with this information the accuracy of the numerical methods is largely increased. In two-dimensions the position of the critical point of some homogeneous models can be deduced from duality relations[6]. The same method works for a random bond Ising model on the square lattice, where the two types of bonds are distributed randomly with equal probability[7],[8]. For periodic Ising models on the square lattice with unit cells of size $m \times n$ an exact relation for the critical temperature can be derived with the use of the Pfaffian method[9] or the transfer matrix technique[10]. This relation is given by a matrix equation which involves $m$-product of non-commuting $2^{n-1} \times 2^{n-1}$ matrices. Therefore in the non-periodic limit $m \rightarrow \infty$ the critical temperature is explicitly known only for $n=1$, i.e. for simple layered systems[11].

The homogeneous Ising model has also been solved exactly on triangular and honeycomb lattices for a long time[12],[13]. The partition function of the models on these hexagonal lattices are related through a star-triangle transformation[6]. A repeated use of this mapping is the basis of an exact renormalisation group transformation by Hilhorst, Schick and van Leeuwen [14], which method has also been used to study the surface critical behaviour of layered models[15]. With this method one can also obtain numerical estimates on the critical point of layered Ising models on hexagonal lattices, however up to now the exact position of the phase transition temperature is not known analytically.

In the present paper we consider this problem and derive an explicit expression for the critical temperature for arbitrary distribution of the couplings both on the triangular and honeycomb lattices. In the calculation one may directly generalize Houtappel's solution 
for the homogeneous model[12]. We will, however, proceed on a simpler way and utilize the correspondences between the square and hexagonal lattices. Let us consider a square lattice, where every second horizontal couplings have a special strength, denoted by $K$ (Fig 1a). Taking the limit $K \rightarrow \infty$ the lattice becomes equivalent to the triangular lattice, whereas for $K=0$ one is left with a honeycomb lattice. Thus the exact solution of layered triangular and honeycomb lattice Ising models can be obtained from the corresponding results of periodic models with a $m \times 2$ unit cell on the square lattice.

The problem of periodic Ising models on the square lattice has been solved analytically first by Hamm[9] with the Pfaffian method and later by Hoever[10] with the transfer matrix technique. Here we use results and notations of the second method.

Let us consider $M \times N$ Ising spins on a square lattice. As indicated on Fig.1a the vertical couplings $K_{j}^{k}(j=1,2, \ldots M, k=1,2, \ldots N)$ are given by:

$$
K_{j}^{k}= \begin{cases}K & k+j=\text { odd } \\ K_{j} & k+j=\text { even }\end{cases}
$$

whereas the horizontal couplings are the same within one column:

$$
\tilde{K}_{j}^{k}=\tilde{K}_{j}
$$

The model is periodic in the horizontal direction: $K_{j+m}=K_{j}, \tilde{K}_{j+m}=\tilde{K}_{j}$. In the following we consider only purely ferromagnetic models, i.e. $K_{j}>0, \tilde{K}_{j}>0$. As mentioned before $K \rightarrow \infty$ and $K=0$ correspond to the triangular and honeycomb lattices, respectively. Introducing the notations

$$
\tilde{x}_{j}=\sinh \left(2 \tilde{\mathrm{K}}_{\mathrm{j}}\right)=\left(\sinh \left(2 \mathrm{~L}_{\mathrm{j}}\right)\right)^{-1}
$$

one can write the partition sum of the model as[10],[11]:

$$
Z=\prod_{j=1}^{m}\left(2 \tilde{x}_{j}\right)^{\frac{N M}{2 m}} \operatorname{trace} T
$$

where $T$ is the transfer matrix:

$$
T=\prod_{j=1}^{m} T(j), \quad T(j)=T_{1}(j) T_{2}(j)
$$

Here

$$
T_{1}(j)=\exp \left(L_{j} \sum_{k=1}^{N} \sigma_{k}^{x}\right)
$$


whereas $T_{2}(j)$ is different for odd and even indices:

$$
\begin{aligned}
T_{2}(2 l-1) & =\exp \left(\sum_{k=1}^{N / 2} K_{2 l-1} \sigma_{2 k-1}^{z} \sigma_{2 k}^{z}+K \sum_{k=1}^{N / 2} \sigma_{2 k}^{z} \sigma_{2 k+1}^{z}\right) \\
T_{2}(2 l) & =\exp \left(K \sum_{k=1}^{N / 2} \sigma_{2 k-1}^{z} \sigma_{2 k}^{z}+\sum_{k=1}^{N / 2} K_{2 l} \sigma_{2 k}^{z} \sigma_{2 k+1}^{z}\right)
\end{aligned}
$$

and $\sigma_{k}^{x}, \sigma_{k}^{z}$ are Pauli matrices at site $k$.

After performing the Jordan-Wigner transformation the transfer matrix is expressed in terms of fermion operators, which in Fourier representation reads as:

$$
T=\prod_{0 \leq q<\pi / 2} T(q), \quad T(q)=\prod_{j=1}^{m} T_{j}(q), \quad T(j)=\prod_{0 \leq q<\pi / 2} T_{j}(q)
$$

where the allowed values of $q$ are equidistantly spaced by $2 \pi / M$. Denoting the leading eigenvalue of the transfer operator $T(q)$ by $\exp (\lambda(q))$, the free energy per spin $f$ is given by:

$$
-\beta f=\frac{1}{2 m} \sum_{j=1}^{m} \ln \left(2 \tilde{x}_{j}\right)+\frac{1}{2 \pi m} \int_{0}^{\pi / 2} \operatorname{dq} \lambda(\mathrm{q})
$$

At the phase transition temperature the free energy is singular, which is a consequence of the singularity in $\lambda(q)$ at the border of the Brilliouin-zone, i.e. at $q_{c}=0$. Our aim in the following is to determine the position of this singularity, i.e. to calculate the critical temperature of the system.

First, following Ref[10] we introduce the parity operator

$$
P=\sigma_{1}^{x} \sigma_{2}^{x}
$$

which commute with the transfer operator $T(q)$, thus $T^{P}(q)$ acts only on a subspace with parity eigenvalue $P= \pm 1$. For sufficiently high temperatures the largest eigenvalue $\lambda\left(q_{c}\right)$ is in the sector with $P=1$, whereas for $T=0$ it is usually in the sector with $P=-1$. $\dagger$ Since the jump in the largest eigenvalue from one sector to the other can not be done analytically there is a phase transition point in the system at a temperature $T_{c}$ where

$$
\lambda^{+}\left(q_{c}, T_{c}\right)=\lambda^{-}\left(q_{c}, T_{c}\right)
$$

$\dagger$ It could happen in frustrated systems with ferro- and antiferromagnetic couplings, that at any finite temperatures $\lambda^{+}\left(q_{c}\right)>\lambda^{-}\left(q_{c}\right)$. Then the free energy is analytical and there is no phase transition in the system. 
At the border of the Brillouin-zone the transfer operator $T_{j}^{P}\left(q_{c}\right)$ can be easily evaluated. It is a $2 \times 2$ matrix given by[10]:

$$
T_{j}^{P}\left(q_{c}\right)=\left(\begin{array}{cc}
C_{j} E_{j} & S_{j} E_{j} \\
S_{j} E_{j}^{-1} & C_{j} E_{j}^{-1}
\end{array}\right)
$$

where $C_{j}=\cosh \left[\mathrm{L}_{\mathrm{j}}(1+\mathrm{P})\right], S_{j}=\sinh \left[\mathrm{L}_{\mathrm{j}}(1+\mathrm{P})\right]$ and $E_{j}=\exp \left[\left(K-K_{j} P\right)(-P)^{j}\right]$. Thus the transfer operator is different for odd and even rows.

In the following we consider the triangular lattice, which corresponds to $K \rightarrow \infty$. In this limit the transfer operator has a simple structure. Keeping terms in leading exponential order the product of two consequtive transfer operators is given by:

$$
\prod_{j^{\prime}=j}^{j+1} T_{j^{\prime}}^{+}\left(q_{c}\right)=\left[\prod_{j^{\prime}=j}^{j+1} \exp (K) \exp \left(-K_{j^{\prime}}\right) \sinh \left(2 \mathrm{~L}_{\mathrm{j}^{\prime}}\right)\right]\left(\begin{array}{cc}
0 & 0 \\
\operatorname{coth}\left(2 \mathrm{~L}_{\mathrm{j}+1}\right) & 1
\end{array}\right)+O(1)
$$

and

$$
\prod_{j^{\prime}=j}^{j+1} T_{j^{\prime}}^{-}\left(q_{c}\right)=\left[\prod_{j^{\prime}=j}^{j+1} \exp (K) \exp \left(K_{j^{\prime}}\right)\right]\left(\begin{array}{ll}
1 & 0 \\
0 & 0
\end{array}\right)+O(1)
$$

for the $P=1$ and $P=-1$ sectors, respectively. Similarly, in this limit any product of the $T_{j}^{P}\left(q_{c}\right)$-s is given in a simple form and the eigenvalues $\lambda^{ \pm}\left(q_{c}\right)$ can be expressed explicitly. Then from eq(10) one obtains the criticality condition as:

$$
\prod_{j=1}^{m} \sinh \left(2 \tilde{\mathrm{K}}_{\mathrm{j}}\right) \exp \left(2 \mathrm{~K}_{\mathrm{j}}\right)=1
$$

This remarkably simple formula is similar to the critical point condition obtained on the square lattice for a simple layered model, i.e. for $n=1[11]$. It is easy to check that eq(13) contains, as a special case, the criticality condition for the homogeneous system[12].

Next we turn to study the critical point condition for the honeycomb lattice. Let us first consider that system which is obtained from the square lattice on Fig 1a by removing the wavy bonds. The transfer operator $T_{j}^{P}\left(q_{c}\right)$ of the system, given in eq(11) with $K=0$, is still a $2 \times 2$ matrix, i.e. not essentially simpler than for the original problem on the square lattice. Therefor there is no explicit relation, similar to eq(13), for the critical point of the layered honeycomb lattice Ising model which corresponds to Fig 1a with vanishing $K$ bonds. However, if the layered structure is that shown on Fig 1c, i.e. the vanishing bonds on the corresponding square lattice are horizontal, then one can derive a similar 
relation to eq(13). Here, instead of repeating steps of the previous calculation we make use the star-triangle mapping[6] and transform the result in eq(13). This transformation maps the honeycomb lattice with bonds $p_{j}, \tilde{p}_{j}$ onto a triangular one with lattice constants $K_{j}$ and $\tilde{K}_{j}$, such that

$$
\exp \left(-4 K_{j}\right)=\frac{\cosh ^{2}(\mathrm{p})}{\cosh \left(\mathrm{p}_{\mathrm{j}}+2 \tilde{\mathrm{p}}_{\mathrm{j}}\right) \cosh \left(\mathrm{p}_{\mathrm{j}}-2 \tilde{\mathrm{p}}_{\mathrm{j}}\right)} \quad, \quad \exp \left(-4 \tilde{K}_{j}\right)=\frac{\cosh \left(\mathrm{p}_{\mathrm{j}}+2 \tilde{\mathrm{p}}_{\mathrm{j}}\right)}{\cosh \left(\mathrm{p}_{\mathrm{j}}-2 \tilde{\mathrm{p}}_{\mathrm{j}}\right)}
$$

Substituting these relations into eq(13) one obtains the criticality condition for the layered honeycomb lattice:

$$
\prod_{j=1}^{m} \sinh \left(2 \tilde{\mathrm{p}}_{\mathrm{j}}\right) \tanh \left(\mathrm{p}_{\mathrm{j}}\right)=1
$$

As a special case this equation contains the criticality condition for the homogeneous Ising model on the honeycomb lattice[12].

We note that relations in eqs(13) and (15) have been numerically verified by the recursion method of Hilhorst and van Leeuwen[15]. Results on the surface critical behaviour of aperiodic Ising models, obtained by that method will be presented in a separate publication $[16]$.

Acknowledgement: This work has been supported by the Hungarian National Research Fund under grant No. OTKA TO12830. 


\section{References:}

[1] J.M. Luck, J. Stat. Phys. 72, 417 (1993)

[2] F. Iglói, J. Phys. A26, L703 (1993)

[3] L. Turban, F. Iglói and B. Berche, Phys. Rev. B49, 12695 (1994)

[4] B. Berche, P.E. Berche, M. Henkel, F. Iglói, P. Lajkó, S. Morgan and L. Turban, J. Phys. A (in print)

[5] A.B. Harris, J. Phys. C7, 1671 (1974)

[6] I. Syozi, in Phase Transitions and Critical Phenomena, Vol.1 edited by: C. Domb and M.S. Green, (London:Academic) (1972)

[7] R. Fisch, J. Stat. Phys. 18, 111 (1978)

[8] B. Derrida, B.W. Southern and D. Stauffer, J. Phys.(Paris) 48, 335 (1987)

[9] J.R. Hamm, Phys. Rev. B15, 5391 (1977)

[10] P. Hoever, Z. Phys. B48, 137 (1982)

[11] W.F. Wolff, P. Hoever and J. Zittartz, Z. Phys. B42, 259 (1981)

[12] R.M.F. Houtappel, Physica 16, 425 (1950)

[13] H.N.V. Temperley, in Phase Transitions and Critical Phenomena, Vol.1 edited by: C. Domb and M.S. Green, (London:Academic) (1972)

[14] H.J. Hilhorst, M. Schick and J.M.J. van Leeuwen, Phys. Rev. 19, 2749 (1979)

[15] H.J. Hilhorst and J.M.J. van Leeuwen, Phys. Rev. Lett. 47, 1188 (1981)

[16] F. Iglói and P. Lajkó (to be published) 


\section{Figure Captions:}

Fig 1 Connection between the square (a) triangular (b) and honeycomb (c) lattices. The square lattice with infinitely strong $K$ bonds (denoted by wavy lines) is equivalent to the triangular lattice, which is related to the honeycomb lattice through a star-triangle transformation[6]. 